\title{
Explanatory Unification and the Early Synthesis Anya Plutynski
}

\begin{abstract}
The object of this paper is to reply to Morrison's ([2000]) claim that while 'structural unity' was achieved at the level of the mathematical models of population genetics in the early synthesis, there was explanatory disunity. I argue to the contrary, that the early synthesis effected by the founders of theoretical population genetics was unifying and explanatory both. Defending this requires a reconsideration of Morrison's notion of explanation. In Morrison's view, all and only answers to 'why' questions which include the 'cause or mechanism' for some phenomenon count as explanatory. In my view, mathematical demonstrations that answer 'how possibly' and 'why necessarily' questions may also count as explanatory. The authors of the synthesis explained how evolution was possible on a Mendelian system of inheritance, answered skepticism about the sufficiency of selection, and thus explained why and how a Darwinian research program was warranted. While today we take many of these claims as obvious, they required argument, and part of the explanatory work of the formal sciences is providing such arguments. Surely, Fisher and Wright had competing views as to the optimal means of generating adaptation. Nevertheless, they had common opponents and a common unifying and explanatory goal that their mathematical demonstrations served.

1 Introduction: Morrison's challenge

2 Fisher $v$. Wright revisited

3 The early synthesis

4 Conclusion: unification and explanation reconciled
\end{abstract}

\section{Introduction: Morrison's challenge}

Morrison ([2000]) has recently argued that unification and explanation are often at odds in science. Using a series of examples from the history of the physical and biological sciences, she argues that there is often a trade-off between systematic unity at the level of mathematical theory and genuine explanation in science. I will counter Morrison's argument, considering one example from the history of science that she uses as a case in point: the disagreements between Fisher and Wright over the major mode of 
evolutionary change. While Morrison is surely correct that these two authors of the synthesis disagreed on a number of points, I will argue that this is not a case where unification and explanation are entirely at odds. My disagreement with her treatment of this case hinges upon a more general disagreement over scientific explanation.

Part of Morrison's project is to refute Kitcher's ([1989]) view of explanation. He argues that to give an explanation is to show how the object of explanation belongs to a class of phenomena that are derivable from a common argument pattern. Explanations, on this view, serve to organize and systematize the phenomena; the aim of scientific explanation is to show how a diversity of phenomena can be brought under a single theoretical framework. The various sciences advance our understanding of nature by supplying increasingly unified accounts of data and related empirical generalizations.

Morrison argues that derivation is not explanation. For instance, while Newton's laws are unifying in that they enable us to derive the motions of the planets and the trajectories of comets through space, they fail to be explanatory (according to Morrison) in that the mechanism, gravity, is left unexplained. As she says:

[O]ne of the most striking features of the Principia is its move away from explanations of planetary motions in terms of mechanical causes. Instead, the mathematical form of force is highlighted; the planetary ellipses discovered by Kepler are 'explained' in terms of a mathematical description of the force that produces those motions. Of course, the inverse-square law of gravitational attraction explains why the planets move in the way they do, but there is no explanation of how this gravitational force acts on bodies, (how it is transported), nor is there any account of its causal properties.

What this suggests is that explanation and unification may not be as closely related as has typically been thought; unity is possible without a satisfactory level of explanatory power (Morrison [2000], p. 4).

There are two ways of interpreting the above passage. Morrison could simply be suggesting that Newton's theory did not give a complete explanation. The inverse-square law explains; but the mechanism by which the law itself operates is not explained. But of course, a theory may explain even if it leaves some issues open to future investigation. In this sense, no scientific explanation is ever complete. If this is what she means, then the point would not be contentious. It seems Morrison wishes to make a stronger point. On her view, accounts of the phenomena that depend entirely upon mathematical derivation are not explanatory, they do not provide 'satisfactory' explanations. Newton formed no hypothesis about how force acts at a distance; force at a distance is, in Einstein's words, 'spooky'. So, Newton's was not a 'satisfactory' explanation. 
True explanations, Morrison argues, ought to provide us with the how and why at work in any particular pattern or process we attempt to explain. For something to count as an explanation, for Morrison, it must provide 'the machinery, or causal behavior, of a particular system', 'explanatory detail' and 'the mechanism that explains why, but more importantly how, a certain process takes place' (Morrison [2000], pp. 3-5). Mathematical theories may provide the kind of generality that makes unification possible. However, these sorts of unifying theories, Morrison says, often sacrifice explanatory detail-just that 'machinery' which makes an explanation explanatory.

It is easy to feel the pull of this claim. However satisfying a prediction from general laws of the trajectory of a comet through space might be, we may still have genuine cause to wonder: what is this mysterious force of gravity and how does it work? How does force act at a distance?

However, the fact that we may further explain Newton's laws by appeal to the curvature of space, for instance, does not imply that the laws are not explanatory. Whether or not an account of the operation of some factor such as 'gravity' or 'selection' is relevant in some explanatory context depends upon the question under investigation. Often what scientists seek is not the details but the 'big picture'-a theoretical framework and a reason or set of reasons for adopting this framework. Explaining why one such framework or research program is the one we ought to adopt is a very important part of science. Doing so often involves appeal to mathematical argument or demonstration. And we may find such a demonstration or argument persuasive and explanatory, not because it answers 'why' questions concerning particulars (e.g. 'Why did the mercury rise?', 'Why did the ball fall?'), but 'how possibly' or 'why necessarily' questions concerning generalities (e.g. 'Why, for any evolving population, is population size relevant to the distribution of gene frequencies in future generations?'). While such explanations may leave causal mechanisms unaccounted for, they are still explanatory, in that they set out a general framework for understanding how any event that meets some set of initial conditions will proceed.

Morrison's view on what makes an explanation 'satisfactory' thus seems too restrictive. It is not necessarily the case that we fail to explain when we give a mathematical demonstration. Why questions range from the particular to the general, and scientific questions include not only 'why' questions, but also 'how possibly?' and 'why necessarily?'. Scientists explain why skepticism about some phenomenon is unwarranted, or how two theories previously understood to be at odds are in fact consistent. These are the sorts of projects that can be achieved via formal, mathematical analysis. The sorts of mathematical models one considers in the formal sciences are not always concerned with describing 'the mechanism' or 'the cause' of some particular phenomenon, so much as setting out which factors in what combinations 
can be expected to be of significance in nature, given some set of initial conditions. Let us call these 'theoretical explanations'. Lewontin has written, with respect to evolutionary theory, that 'the form of this [population genetic] program is to produce purely analytic results of an "if, then" form that can be used to demarcate the allowable from the unallowable claims of explanation,' and, " "the best to which population geneticists can aspire is a formal structure that sets the limits of allowable explanation and a set of existentially modified claims about what has actually happened in the real history of organisms.' (Lewontin, 1985, pp. 199, 213)". In the formal sciences, the object of an explanation is often simply setting out the conditions on the possibility of some phenomenon coming about. In my view, Morrison has short-changed these special explanatory virtues of the theoretical aspect of the sciences, and of theoretical population genetics in particular. Scientific explanation is explanation not simply of the facts, but also of prohibitions and possibilities.

Some may worry that such an account is too permissive; it seems to be an 'anything goes' account, according to which any 'possible story' could function as an explanation. Surely it's possible that celestial harmonies caused the planets to move in elliptical orbits, but this is not a scientific explanation. 'Delineating the possibilities' is, according to this objection, only to 'have an explanation' (i.e., a possible story), not to 'have an acceptable theory that explains' (i.e., having a story that is more than simply one among many possibilities) (van Fraassen [1980]). This is to misunderstand my claim, however. I am not claiming that scientific explanations are simply possible stories. To have a mathematical model that sets out the conditions for the possibility of some event in nature is not simply to 'have an explanation', since the object is not to propose any conceivable explanation for some particular event. Rather, the object of formal analysis and demonstration in the sciences is to delineate the conditions of adequacy of any explanatory story for some domain. The object of such a delineation is to consider a set of factors that have been empirically proven to operate in nature (e.g. selection, mutation, etc.) and to show how they operate in conjunction for any system with some set of properties (e.g. heritable variation in fitness), given some set of initial conditions (e.g. population size, structure, etc.). This may be done without committing to any one interpretation of the causal basis (the "how and why'), for example of selection or mutation in some particular population.

Consider the following example. For any population of organisms with heritable variation in fitness, in populations of very small size, drift, or sampling error will govern changes in genetic constitution to a greater extent than selection. In larger populations and over the long term, even a very small difference in fitness between organisms possessing genotype $x$ and genotype $y$ may yield dramatic changes in the constitution of the population. This particular generalization is reducible to the fact that when we flip a coin biased 
toward heads ten times, we are not as likely to be able to determine that it is a biased coin as when we flip the coin a hundred times (the 'law of large numbers'). The generalization that the effects of drift will override the effects of selection in small populations is not a 'possible story' but a necessary conclusion of the probability theory applied to evolving populations, described as Mendelian systems. It is explanatory in that it answers the question, 'Why must population size play a role in any evolving population (so described)?' So, by 'delineation of the possible', Lewontin does not mean the logically possible, but rather the biologically possible.

\section{Fisher v. Wright revisited}

In the final chapter of her book, Morrison appeals to disagreements between two theoretical population geneticists, R. A. Fisher and Sewall Wright, as a case in point for her claim that unification and explanation are often at odds in science. Morrison's claim is the following. While the founders of this discipline could agree upon the mathematics necessary to represent evolving populations, they fundamentally disagreed on the explanation of 'how selection acted in populations'. According to Morrison:

\footnotetext{
Structural unity achieved by the employment of mathematical methods [. . .] was accompanied by striking disunity at the levels of both methodology and interpretation of the way in which selection and other factors operated [. . .]. So although Fisher and Wright were interested in showing quantitatively how natural selection could operate under certain conditions, the unification was not accompanied by one consistent explanation about how that process actually took place. (Morrison [2000], p. 212)
}

Wright and Fisher agreed on a quantitative way of representing evolving populations that unified Darwinian selection and Mendelian genetics, but, according to Morrison, they disagreed on the qualitative story of how selection operated in populations. In this case we have mathematical unity, but explanatory disunity.

Morrison does an elegant job documenting Fisher and Wright's methodological differences and differences in mathematical technique. However, Fisher and Wright's argument was not over the mechanisms leading to selection's successful operation. Rather, they disagreed on the balance of factors of greatest significance in generating adaptation. On the one hand, Fisher thought that mass selection was the main way in which adaptation came about. Fisher argued that the factor of greatest significance in generating adaptation was selection. On the other hand, Wright believed that a combination of isolation, drift, and intra- and inter-demic selection was both the most effective way of generating adaptation, and a process that operated frequently in nature. Wright argued that adaptation required a 'balance' of factors in 
combination; the relative significance of selection in generating adaptation was not as much as isolation, drift and selection in combination. Morrison calls this a disagreement about 'the successful operation of selection'. But the above is, in my view, better described as a relative significance controversy.

At the root of this debate, however, was not the explanation of adaptation, but the characterization of the problem of adaptation in the first place. In other words, Wright and Fisher were trying to answer different questions. Their differences depended upon their views on the relative significance of genetic interaction and population structure. Some historical context is necessary to understand this point.

Wright had been a laboratory scientist before he turned to the problem of mathematical population genetics. He used guinea pigs as a model system to investigate the inheritance of coat color in mammals - a complex problem that forced him to think not only about genes as simple factors, but the physiology of genetics: how genes were expressed and interrelated. Wright's experience in the lab deeply influenced his conception of the problem of adaptive evolution. Since adaptation was, in Wright's view, a product of gene systems, or combinations of genes that were functionally interrelated, the problem of adaptive evolution was very specific: how is it possible for such highly adapted systems of genes to become dissociated and to reform into a new, more favorable combination? Wright wrote ([1932]) that 'The problem of evolution as I see it is that of a mechanism by which the species may continually find its way from lower to higher peaks in such a field.'

Wright here appeals to his ([1932]) metaphor of 'rugged adaptive landscapes'. A rugged adaptive landscape is a surface in a multidimensional space that represents the mean fitness of the population as a function of gamete (or allele) frequencies. This surface has many peaks and valleys corresponding to different adaptive and maladaptive population states. The population is imagined as a point on the surface that is driven by selection uphill but that can get stuck on a local peak. The problem of adaptation was thus the problem of how populations of interbreeding organisms could shift from one highly adaptive set of gene combinations to another-how they could 'traverse' adaptive valleys, or move from a sub-optimal 'adaptive peak' to an optimal one. Wright assumed that a population's present state was adaptive, in the sense that all the genes in the population were in 'balance'. Any novel gene would force the population down into an adaptive valley. What Wright saw as the problem for evolution was thus contingent upon his presuppositions about gene interaction and adaptation.

Fisher did not share these presuppositions. He argued, in correspondence with Wright (cited in Provine [1986]), that the metaphor of the adaptive landscape was misconceived. It was better, in Fisher's view, to view the field of gene combinations as a multidimensional space. Populations could increase 
their average fitness along any of several possible trajectories. According to Fisher, the problem of adaptive evolution was simply the problem of how to create and maintain enough variation in natural populations for selection to act upon. As long as there was sufficient additive variance in fitness, evolution would proceed necessarily (indeed, by definition, according to the fundamental theorem of natural selection). In Fisher's view, the constraints on evolving populations were not so restrictive that selection alone could not be responsible for a population evolving from a sub-optimal to a more adaptive state. ${ }^{1}$ Fisher was aware that genes had interactive effects (see his [1918] and [1922]), but his view was that these did not prevent populations from becoming more fit. In fact, the interactive effects of genes could overpower deleterious mutations via 'Modifier' genes. Fisher did not offer a qualitative theory of evolution akin to Wright's shifting balance theory, because Fisher did not share Wright's conception of the problem of adaptive evolution. So no such theory was, in his view, necessary. Simple mass selection was, he thought, sufficient for adaptive evolution.

The object of explanation was different for Fisher and for Wright, then, in two senses. First, the preconceptions they had about the genetic structure of populations of organisms in nature were fundamentally different. Second, in part as a result of this, the problems of evolution, those that they believed most required solving, were different. Morrison concludes that their disagreements were over how to explain the 'successful operation' of selection. While I concede to Morrison that Wright and Fisher disagreed, I would suggest that it is better to view their differences as resting upon their different conceptions of the problem of adaptive evolution. It was not the 'successful operation of selection' they disagreed upon so much as the relative significance of selection versus drift, given their different background assumptions about genetic interaction and population structure. For Wright, the most pressing question for evolutionary biology was, 'By what mechanism may a species continually find its way from lower to higher peaks in the adaptive landscape?' For Fisher, the most pressing question for evolution was, 'How is there sufficient variation for selection to act upon?'

This point to one side, let us examine the more general moral which Morrison attempts to draw from this case. Morrison claims that explanation and unification were at odds in the case of the early synthesis. While Morrison is correct that Wright and Fisher disagreed on the factors of greatest significance in generating adaptation, I suggest that this observation by itself does not support her more general conclusion. Morrison's

1 Interestingly, subsequent work by theoretical population geneticists has supported Fisher's views. Gavrilets ([1999]) argues, for example, that Wright's metaphor should be replaced with a 'holey' landscape. 
characterization of the explanatory work of the authors of the synthesis presupposes that all and only explanations which give the complete causal story of how evolution proceeds are satisfactory. Morrison writes:

It was possible, using two different quantitative methods, to produce a unification of genetics with Darwinian theory by showing that selection, even in cases in which only small pressures were exerted, operated in Mendelian populations. Yet, despite agreement on that basic unifying principle, there was significant disagreement at the explanatory level regarding how the evolutionary process actually took place. ([2000], p. 227)

And again,

Theoretical agreement on unification of genetics with evolutionary theory could not have been achieved if the theorists had been required to supply the explanatory details about how and why selection operated in particular types of populations. ([Ibid.], p. 229)

Morrison begs the question against the unificationist in the above passages. A great deal hangs upon her characterization of the 'explanatory level'. If what counts as the 'explanatory level' is only explanations of how the evolutionary process actually takes place, then Morrison has won her point. However, in my view, science aims to explain not only what is actual, but also what is possible and necessary. For instance, agreement on a synthesis of Darwinism and Mendelism was an explanatory feat; it required a mathematical demonstration of how and why the two might be viewed as compatible. It seems too parsimonious to claim that such a demonstration is not explanatory. Explaining 'how and why selection operated in particular types of populations' is certainly part of the work of evolutionary theory. However, it is not all that evolutionary theory explains. Below, I elaborate upon the ways in which theory may serve as explanatory which do not concern the particular or the merely actual.

\section{The early synthesis}

Morrison's thesis is that unification and explanation are often at odds in science. In Morrison's view, an explanation is satisfactory when it identifies the causal mechanism at work in generating some event or state of affairs in the world. Morrison seems to be supporting the view, originally due to Salmon, that an explanation provides a causal story, or 'to give scientific explanations is to show how events [. . .] fit into the causal structure of the world.' However, I think a case can be made that the architects of the early synthesis did indeed provide explanations, but these explanations were of a different sort than the 'causal story' explanations favored by Salmon. Rather, they were explanations of a 'how possibly' and 'how necessarily' sort, discussed above. I will turn Morrison's example on its head, and argue that, in fact, theoretical population genetics was both unifying and explanatory. 
Despite the many subtle and intriguing differences and disagreements among its founders about which questions are of greatest significance and about the optimal means of generating adaptation, theoretical population geneticists in the 1920s and 30s did provide a unified explanation of how Darwinian evolution is possible on a Mendelian system of inheritance. In addition, Fisher, Haldane and Wright demonstrated, using relatively simple mathematical models, that:

1. A very weak selective advantage is sufficient to fix an allele in relatively few generations on an evolutionary time-scale. This sufficiency of selection argument effectively ruled out competing explanations of evolution (mutationist, neo-Lamarckian), as I will explain below.

2. The rates of mutation would not be sufficient to outdistance the force of selection acting in a population. This ruled out 'orthogenesis'-or the idea of mutational pressure driving evolution.

3. In very small populations, selection will be counterbalanced by the random fixation of alleles, or 'genetic drift'. In other words, the effectiveness of selection versus drift is relative to effective population size.

4. An unfavorable, even lethal, recessive mutation will stabilize itself at low frequency, but not disappear from a population. This result is often cited to explain why eugenics would be a slow and inefficient process if directed at homozygotes alone.

Such proofs of possibility are not insignificant in the history of science. They set out the boundary conditions on the range of possible processes that can generate change in a system; setting out the conditions in this way is explanatory in that it yields a quantitative understanding of the 'prohibitions and possibilities' for evolution in any population. The sort of understanding yielded by these mathematical demonstrations is akin to that described by Salmon as 'Weltanschauung'-style understanding, as opposed to 'black box' causal stories. At the time that Fisher and Wright developed these models, there was no systematic account of how the different factors of selection, mutation, migration, drift and assortative mating could generate changes in a population. This work not only provided a unified mathematical way of picturing evolutionary change, but also demonstrated how it was possible for selection to generate the vast diversity that seemed so implausibly created by minor selective advantages offered by .4 centimeter of beak length or carapace width.

First, a caveat: I wish to be clear here about this claim of the sufficiency of selection. I am not claiming that Fisher, Haldane and Wright demonstrated that selection is the only factor in evolution. Rather, they showed that it was possible for selection acting on small, continuous variation to have a significant effect in a relatively short time. By way of making this intuitive, 
imagine the following figure: let the y-axis represent generations, and the $\mathrm{x}$-axis represent proportion of individuals in a population possessing some allele, with a selection coefficient of only .01. Or, the relative advantage in terms of passing on offspring of some individual possessing such an allele is only $1 \%$. The curve that describes the replacement of other alleles by this allele is a logistic curve; the gene will spread very slowly at first, but eventually 'take off' and 'fix' in the population. This is because the effect is multiplicative, rather than additive.

This is the first aspect of what I meant by the 'sufficiency of selection' above: that it is simply possible for small selection coefficients to have a large effect. The second aspect involves an implicit appeal to parsimony: the claim that selection is sufficient to undermine the necessity of appealing to competing mechanisms of evolution. Inheritance of acquired characters, mutationism, orthogenesis and other hypothetical mechanisms of evolution were no longer necessary for the evolutionary process. The mathematical models of theoretical population genetics were capable of generating the plausibility arguments necessary to give biologists good reason to pursue a Darwinian research program. Such prudential arguments are an important part of the explanatory work of science. Note, however, that such arguments do not appeal to 'causal details' of how much selection operated in the determination of this or that trait. This would simply not be relevant.

Population genetics defined which factors are key for evolution, which factors may be ruled out, and how the various parameters of evolution within a population are interrelated. It defined the realm of what is possible, and distinguished it from what is implausible. Moreover, it gave evolutionists a systematic way of representing evolutionary change in populations on a Mendelian system of inheritance, and defined how the different parameters, or mechanisms in evolution, constrained one another. This was explanatory in the following way. It provided a general framework for thinking about evolution, which pulled together what were understood to be competing research traditions under a single common framework, demonstrating that they are not only compatible, but complementary. In sum, population genetics served to define and delineate which processes are sufficient for evolution, effectively reducing the number we need to take as brute: selection, mutation, assortative mating, migration and drift. Prior to the 1920s and 30 s, it was an open question how evolution proceeds, and whether it was necessary to invoke Lamarckian or mutationist mechanisms to supplement selection. Attention to the historical context immediately preceding the development of theoretical population genetics demonstrates how this work served as explanatory.

In 1907, Vernon Kellogg published a state of the union address on the issues yet to be solved in evolutionary biology, entitled Evolution and 
Animal Life. He quoted Henry Fairfield Osborn as saying 'the original Darwinian factor, or Neo-Darwinism, offers an inadequate explanation of evolution' (Kellogg [1908], p. 374). And he added this comment:

Our present plight seems to be exactly this: we cannot explain to any general satisfaction species forming and evolution without the help of some Lamarckian or Eimerian factor; and on the other hand, we cannot assume the actuality of any such factor in the light of our present knowledge of heredity. ([Ibid.], p. 374)

These views represented the majority view before Fisher, Haldane and Wright developed their mathematical models. Many biologists were sympathetic to Darwinism, but found it inadequate, in that it was difficult to understand how selection alone could generate such diversity. Fisher, Haldane and Wright were able to give proofs of this possibility.

Explaining what is possible, and, moreover, ruling out what is implausible, is part of the dialectic of the advancement of science, and is what the important explanatory work of mathematical theories such as theoretical population genetics consists in.

\section{Conclusion: unification and explanation reconciled}

Now I will return to the more general philosophical concern that motivated this paper: the relation between explanation and unification. Morrison's claim was that 'explanation and unification are often at odds in science,' and used the disagreements between Fisher and Wright as a case in point. My argument has been that Fisher and Wright's disagreements do not support her point, but rather provide an example of the contrary. Using mathematical models, Haldane, Wright and Fisher described the conditions for, and constraints on, a general theory of evolution. They provided general theoretical demonstrations of, for instance, the effectiveness of selection and the constraints provided by population size that make no reference to the causal machinery at work in any particular evolving lineage (e.g. ecological conditions, etc.). In Kitcher's words, 'explanations need not, and sometimes should not, deliver information about the causal history of a particular occurrence.' And science may advance our understanding of nature by 'providing information about constraints on causal processes' ([1989], p. 427 and p. 417, my emphasis).

Morrison's view seems to be that the causal view trumps Kitcher's unificationist model; correct or complete explanations, in her view, must appeal to causes. However, I don't see the 'bottom-up' and 'top-down' approaches to explanation as, prima facie, at odds. I take my cue from Salmon ([1984]), who has suggested that there be 'rapprochement' between two competing views. 
Salmon distinguishes two types of explanation, explanation ${ }_{1}$ and explanation $_{2}$, which he roughly aligns with Kitcher's earlier distinction between what he called 'bottom-up' and 'top-down' explanations. The first sort of explanation is when we give a causal story, for instance, in terms of forces acting on some part of a physical system. His example is the case of the friendly physicist in an airplane. He asks the boy sitting across the aisle holding a helium-filled balloon what he thought the balloon might do when the airplane accelerated rapidly for takeoff. After some consideration, the boy suggested that the balloon would move toward the back of the cabin. To the contrary, the physicist wagered that it would move to the front. $\mathrm{He}$ was correct, and won a complimentary bottle of scotch from a bewildered flight attendant. Salmon ([1990], p. 11) describes two explanations of the phenomena: first, as the plane accelerates, the rear wall of the cabin exerts a force on the air molecules near the back, which produces a pressure gradient from rear to front. Given that the inertia of the balloon is smaller than that of the air it displaces, the balloon tends to move in the direction of less dense air. This is a causal explanation in terms of the forces exerted on the parts of the physical system. Second, one can appeal to Einstein's principle of equivalence, which says that an acceleration is physically equivalent to a gravitational field. The effect of acceleration of the airplane is the same as that of a gravitational field. Since a helium balloon in air will rise in the earth's gravitational field, so too it will tend to move forward in the air of the cabin in the presence of the aircraft's acceleration. This second explanation is an example of a unificationist or 'top-down'-type explanation, in that it shows how this one case falls under a single argument pattern, or how it fits within a certain theoretical framework.

I wish to argue the following: scientific explanation is not exclusively a 'top-down' or 'bottom-up' affair. I think that the two are complementary; exclusive emphasis on one or the other is misguided. Often, what we are willing to take as acceptable causal stories is contingent upon our best available theory. For instance, in the case of the balloon example above, we no longer appeal to Newton's theory of gravitation, but to Einstein's principle of equivalence. Likewise, in the case of evolutionary biology, biologists today do not appeal to Lamarckian use and disuse or mutational pressure. These sorts of causal mechanisms were rendered unnecessary by the sorts of theoretical arguments offered by population geneticists in the 1920s and 30s.

In other words, scientific explanations may not only tell us why or how some fact in the world is so, they may also rule out unnecessary factors or mechanisms, and define how those mechanisms function and constrain one another. This is exactly what theoretical population genetics does. As a result of these demonstrations, population genetics explained how and why many of the then-live alternatives to Darwinian evolution were no longer viable. 
Neo-Lamarckism, orthogenesis, mutationism and ultra-Darwinism all held that there were ultimate forces of evolution that somehow directed evolution: either internal, in the case of the mutationists, or external, in the case of the Lamarckians. Population geneticists were able to explain how some fundamental force need not direct evolution, either from within or outside of the organism. There was no 'ultimate factor' to evolution.

Now, I need to say a bit here about the view I am not defending: I am not defending the 'unification writ large' view, according to which all the sciences are, or will be, reducible to physics. I am not defending a reduction or elimination of causation, though with Kitcher I think that answering Hume's worries is an extremely difficult project, which has not yet been sufficiently addressed. ${ }^{2}$ Rather, I am suggesting that in the case of theoretical population genetics at least, unification and explanation are not at odds; and indeed, here is a case which brings into question Morrison's thesis. Morrison makes assumptions about explanation: that explanations are always answers to 'why' questions, and that the task of explanation is to supply a causal mechanism. I take it that I have demonstrated how both of these assumptions are questionable. Explanations may also be answers to 'how possibly' questions - where what is proffered in reply is not the 'supply of a causal mechanism', but rather an account of constraints on any causal process.

The sort of explanation that I have attempted to bring attention to here is the sort that directs us toward a unified research program. This may involve appeal to a family of models and model arguments. One important component of the factors that made the evolutionary synthesis possible was the application of statistical methods and mathematical models to the problems of variation, heredity and evolution via natural selection. Fisher, Haldane and Wright developed a heuristic that contributed to the way evolution was conceived; namely, as a genetic process. Their major mode of explanation was theoretical models. They used these mathematical models to show how and why, among other questions, the sex ratio comes to be $1: 1$, and how effective population size affects rate of loss of genetic variation. These models served both to unify the Darwinian perspective with Mendelian genetics and to structure the field of permissible answers to evolutionary questions.

\section{Acknowledgements}

I would like to thank M. Morrison, G. Hatfield, those who attended my session at the 2001 meeting of the International Society for History, 
Philosophy, and Social Studies of Biology, and an anonymous reviewer for their invaluable comments and encouragement.

Department of Philosophy University of Utah

260 S. Central Campus Drive

Salt Lake City, UT 84112

USA

anya.plutynski@philosophy.utah.edu

\section{References}

Fisher, R. A. [1918]: 'The correlation between relatives on the supposition of Mendelian Inheritance', Transaction of the Royal Society of Edinburgh, 52: 399-433.

Fisher, R. A. [1922]: 'On the dominance ratio'. Proceedings of the Royal Society of Edinburgh. 42: 321-41

Gavrilets, S. [1999]: 'A dynamical theory of speciation on holey adaptive landscapes', American Naturalist, 154 (1), pp. 1-22.

Hitchcock, C. [1993]: 'A Generalized Probabilistic Theory of Causal Relevance', Synthese, 97, pp. 335-64.

Kellogg, V. L. [1908]: Darwinism To-day: a Discussion of Present-Day Scientific Selection Theories. London: Bell.

Kitcher, P. [1989]: 'Explanatory Unification and the Causal Structure of the World', in Kitcher, P. and Salmon, W. Scientific Explanation. Vol 13. Minnesota Studies in the Philosophy of Science. Minneapolis: University of Minnesota Press.

Lewontin, R. [1985]: 'What do Population Geneticists Know and How do they Know it?', in Creath, R. and Mainschein, J. (eds.), Biology and Epistemology, Cambridge: Cambridge University Press.

Morrison, M. [2000]: Unifying Scientific Theories: Physical Concepts and Mathematical Structures, Cambridge: Cambridge University Press.

Provine, W. [1971]: The Origins of Theoretical Population Genetics, Chicago: University of Chicago Press.

Provine, W. [1986]: Evolution: Selected Papers by Sewall Wright, University of Chicago Press: Chicago.

Salmon, W. [1984]: Scientific Explanation and the Causal Structure of the World, Princeton: Princeton University Press.

Salmon, W. [1990] "Scientific Explanation: Causation and Unification" in Critica: Revista Hispanoamericana de Filosofia. Vol. XXII, No. 66, Mexico: National University of Mexico. December 1990.

Sewall Wright, R. A. [1931]: 'The Roles of Mutation, Inbreeding, Crossbreeding, and Selection in Evolution', Proceedings of the $6^{\text {th }}$ International Congress in Genetics, $\mathbf{1}$, pp. 356-66. 
Skipper, R. [2002]: 'The Persistence of the R. A. Fisher-Sewall Wright Controversy', Biology and Philosophy, 17 (3), pp. 341-67.

van Fraassen, B. [1980]: The Scientific Image, Oxford: Oxford University Press.

Wright, S. [1932]: 'The Roles of Mutation, Inbreeding, Crossbreeding, and Selection in Evolution', Proceedings of the 6th International Congress in Genetics, 1, pp. 356-66, in Provine, (ed.) Evolution: Selected Papers by Sewall Wright, University of Chicago Press: Chicago. 